Pierre Asselot

\section{El paisaje del vino}

1 Roberto Fernández propone el término "geocultura" para expresa las relaciones de una sociedad con su medio físico y que remiten a una apropiación de la naturaleza. En Fernandez, Roberto, El laboratorio y regionalismo. Editorial Biblioteca Nueva, Madrid, 1998, pp. 185-227, 247-265.
2 VV, AA. La Mouvance, du jardin au territoire, "Geograme", Augustin
Berque, Ed. Ecole d'architecture de L Villette, Paris, 1999, pp. 68.
Las primeras bodegas dedicadas a la explotación comercial de los viñedos eran definidas por un programa preciso y normado y la disponibilidad de ciertos materiales. La representación del cultivo de viñas, relacionado entonces a una situación de territorio organizado productivamente como sistema (la casa patronal, la capilla, el parque, las viñas y las casas de inquilinos) boy más bien aparece ligada a esquemas más sencillos entre un edificio y la vid, que evidentemente centran su atención en la arquitectura de ese edificio. Asselot presenta una visión de esas primeras viñas, planteadas como un lugar de producción más que como generadores programados de identidad.

Palabras clave: Paisaje-viñas, paisajismo-territorio, Llano del Maipo, viñedos.

An exact, regulated brief and the availability of certain materials defined the first wine cellars built to exploit the wineries commercially. The representation of vineyard culture, related in the past to a territory organized productively as a system (mansion house, chapel, park, vineyards and farm workers' houses), now seems to relate to simpler schemes of building and vine, which clearly focuses attention on the architecture of the building. Asselot offers a vision of those first vineyards as a place of production, rather than programmed creators of identity.

Key words Landscape-vineyards, landscape-territory, Llano del Maipo, vineyards.

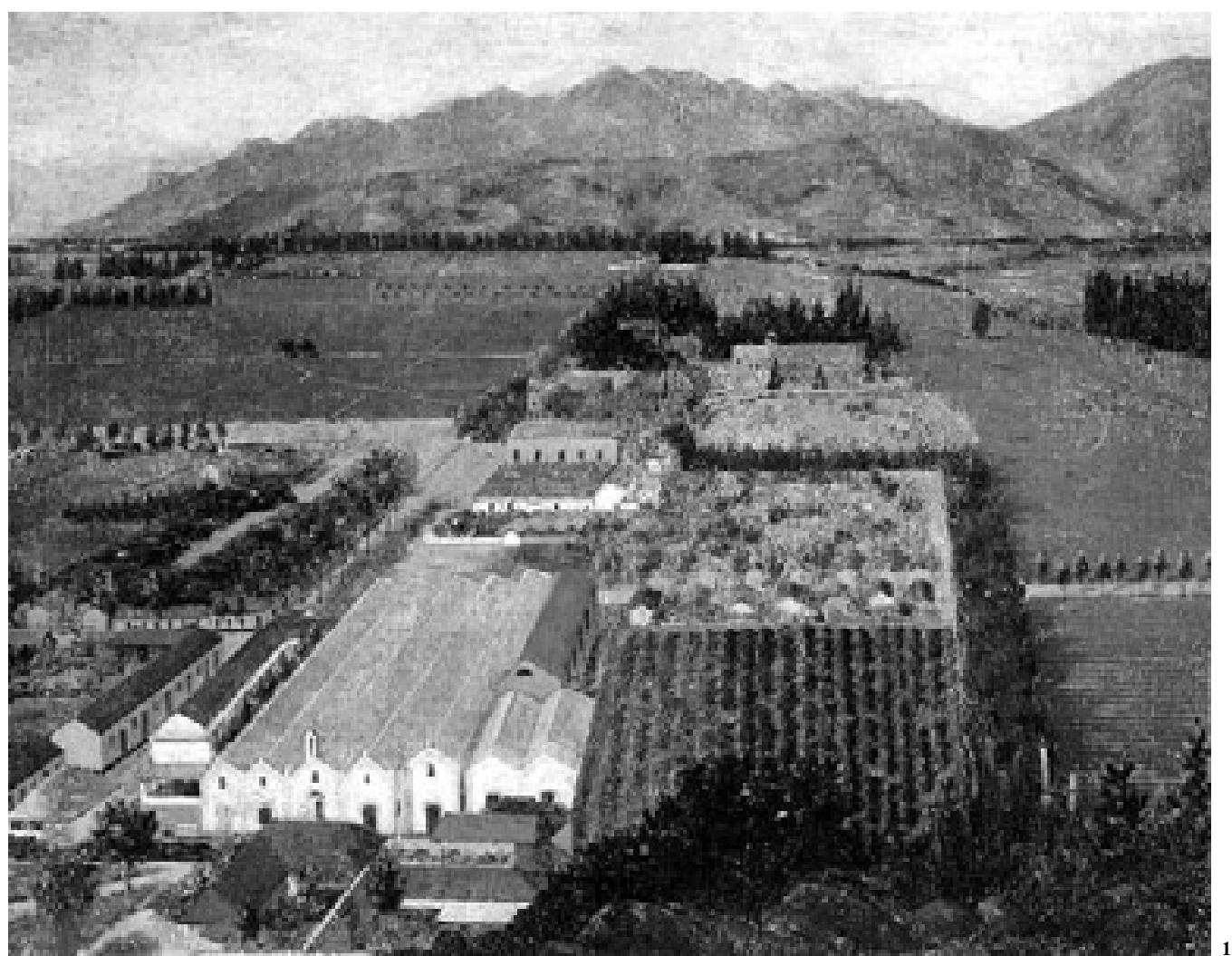

Un punto de vista sobre el territorio y espacio rural en el Llano del Maipo a finales del siglo XIX

\section{Introducción}

A finales del s. XIX la implementación de infraestructuras en los valles centrales y la aparición de nuevas estructuras agrícolas pueden considerarse como un conjunto de acciones que apuntan a valorizar de manera productiva el espacio rural. $\mathrm{La}$ "geocultura" entiende el conjunto de acciones del hombre en una determinada geografía como un aspecto representativo de la construcción de un territorio. Estas acciones implementadas por una cultura permiten la apropiación del espacio físico, produciendo un conjunto de manifestaciones concretas que toman sentido en el marco de esa cultura. Son estas manifestaciones las que Augustin Berque llama "geogramas", motivos eco-simbólicos que configuran el paisaje.

Se puede entonces considerar, por un lado, las transformaciones del Llano del Maipo, en el marco de la reforma de la vitivinicultura al final del siglo XIX, como la construcción de un territorio del vino, y por otro lado intentar reconocer las manifestaciones de estas transformaciones como los geogramas que configuran el paisaje del vino.

El cuadro que representa la viña Panquehue (Fig.1) ilustra la dualidad de la palabra paisaje que conlleva dos sentidos. Por un lado se llama "paisaje" a una pintura o dibujo que representa una porción de territorio. Igualmente se llama "paisaje" a la porción misma de territorio que se encuentra frente a un observador. La definición de la palabra "paisaje" involucra al objeto de la representación y la representación en sí misma. Lo importante es que la existencia de una representación pictórica de una explotación vitivinícola identificable, como la viña Panquehue, valida la hipótesis de la existencia de un paisaje del vino. Este cuadro es una representación que sitúa al espectador en el punto de vista del pintor que consideró la porción de territorio como un paisaje. Es una representación que da sentido a los elementos que construyen la imagen y permite leerlos como los geogramas del territorio representado.

El presente texto propone reconocer el contexto histórico y geográfico en el cual se constituyó el paisaje del vino, a finales del s. XIX. Para este fin, se desglosó la imagen de este paisaje, identificando las infraestructuras y las nuevas 
1 Pintura de la viña Errázuriz Panquehue, 1896.

Autor desconocido. Tomada

de Le Blanc, Magdalena, $E l$

vino chileno, una geografía

optima, Ocho Libros Editores,

Santiago de Chile, 2000
2 El Llano del Maipo y Santiago

en 1895. Trazado de canales,

ferrocarriles y su relación

con los predios agrícolas de la

periferia (dibujo del autor)

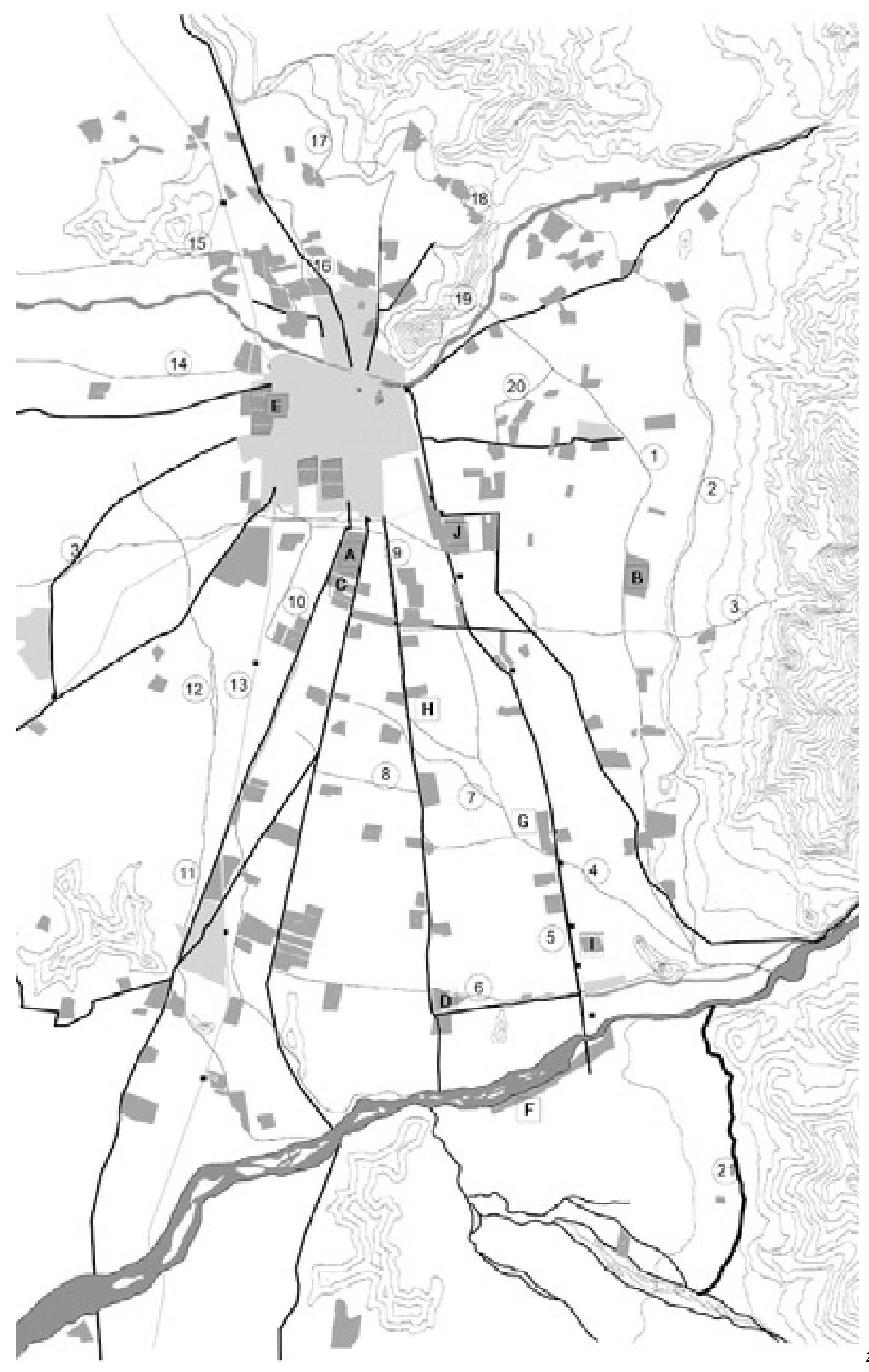

Santiago 1895

Viñedos

Casco Urbano

(1) Canal San Carlos

(2) Canal Las Perdices

(3) Zanjón de La Aguada

(4) Canal San Francisco

(5) Canal San José

(6) Canal San Bernardo

(7) Canal San Gregorio

(8) Canal Cisterna

(9) Canal San Joaquín

(10) Canal Ochagavía

(11) Canal de Lo Espejo

(12) Canal de Ortúzar

(13) Canal de Rulo

(14) Canal de Zapata

(15) Canal de La Punta

(16) Canal de Lo Pinto

(17) Canal de La Pólvora

(18) Canal del Carmen

(19) Canal Santo Domingo

(20) Canal San Migue

(21) Canal La Sirena

A El Llano Subercaseaux

B Cousiño Macul

C Vial

$D$ San José

E Quinta Normal

$F$ Concha y Toro

G Quillayes

$\mathrm{H}$ Victoria

1 San Carlos

J Santa Carolina 
unidades de explotación vinícola, como articuladores de un cuadro coherente.

Contexto histórico y geográfico: la reforma de la vitivinicultura en el valle de Santiago Hasta mediados del siglo XIX la viticultura chilena básicamente producía un tipo de vino que, desde la colonia, se elaboraba de manera artesanal sin particular evolución en los procedimientos de vinificación.

Esta situación dejaba en desventaja a los vinos chilenos frente a los importados desde Europa, de mejor calidad. La desventaja local radicó básicamente en no contar con una elaboración técnicamente apropiada, que permitiera tanto una buena calidad como una adecuada conservación en el tiempo.

La introducción de nuevos cepajes provenientes de Francia y la adaptación de técnicas de producción controladas por enólogos fueron factores que impulsaron la reforma vitivinícola.

Al implementar las técnicas necesarias para la elaboración y conservación de un vino de calidad, la enología tuvo como objetivo garantizar a priori un producto de calidad que buscaba competir en diferentes mercados.

El año 1851 marcó el comienzo de la reforma, cuando Silvestre Ochagavía 3 contrató al enólogo francés, Joseph Bertrand, para la producción de su vino de cepas francesas en las afueras de Santiago. La asociación de propietarios nacionales con técnicos europeos fomentó la incorporación constante de las últimas técnicas en el establecimiento de nuevas viñas.

Pero, a raíz de la anexión de los territorios mineros del norte, la confianza en el crecimiento del mercado interno atenuó la voluntad de competir en el mercado de exportación, y a pesar de disponer con todos los adelantos técnicos necesarios para producir un vino de guarda, la producción fue irregular en calidad, privilegiando la cantidad.

Con el fin de la I Guerra Mundial se selló la reforma de la vitivinicultura. Lógicamente, la crisis salitrera junto con la apatía de los mercados extranjeros provocaron una disminución en la demanda, gatillando una larga crisis de la industria vitivinícola, efecto que fue ampliado por la crisis económica de 1929.

Sin embargo, este ciclo del mundo vitivinícola participó, en un período de ochenta años, en un proceso de transformación del espacio rural $^{4}$. La región central y sus valles ofrecían las condiciones de suelo y clima más favorables para la implantación de las viñas modernizadas y los nuevos cepajes.

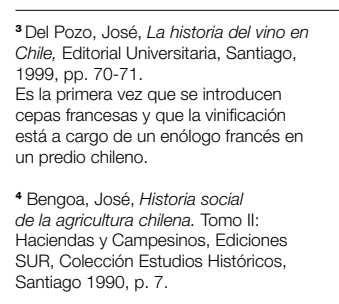

Santiago 1990, p. 7 .
No es de extrañar que la zona agrícola dedicada a la nueva viticultura tuviera como límite norte el río Aconcagua y como límite sur el valle de Curicó. Entre estos dos valles se encuentra el valle del Maipo, en el cual se concentró una alta cantidad de nuevas viñas en proximidad a la ciudad de Santiago y en particular en el sector del Llano del Maipo.

Tres escalas para un cuadro del vino: Las infraestructuras como telón de fondo Las infraestructuras propias del siglo XIX estuvieron en el origen del desarrollo de las nuevas viñas y provocaron su dinámica. La construcción y el trazado de canales de irrigación y ferrocarriles perfilaron un papel determinante para rentabilizar el espacio rural y definir nuevos territorios para la agricultura y para la vitivinicultura (Fig. 2).

La Asociación de los Canalistas del Maipo ${ }^{5}$ irrigaba alrededor de 100.000 ha de predios agrícolas del valle de Santiago, cuyos accionistas eran propietarios de viñas. En la misma lógica, en 1889 la iniciativa de la construcción del Ferrocarril del Llano del Maipo ${ }^{6}$, que hacía el recorrido de Santiago a Puente Alto, se debe a Domingo Concha y Toro.

En varios casos fueron, entonces, los mismos emprendedores ${ }^{7}$ los que valorizaron el Llano del Maipo y aprovecharon sus infraestructuras para implementar sus viñedos modernizados.

Así, el territorio del vino no sólo se definió como el resultado de operaciones de infraestructuras y de explotaciones vitivinícolas sobre el espacio geográfico, sino que, además, reflejó las relaciones entre el campo y la ciudad, a finales del siglo XIX.

\section{Agrado y producción: características espaciales de las nuevas viñas}

$\mathrm{Si}$ bien los conocimientos de los enólogos permitieron la detección de los mejores terruños ${ }^{8}$, las posibles ubicaciones de las viñas buscaron además beneficiarse de las infraestructuras territoriales.

Las unidades de producción surgidas de la reforma vitivinícola ${ }^{9}$ fueron caracterizadas por una racionalización en su ubicación, organización interna y distribución de los espacios al interior del predio.

En el Llano del Maipo la ubicación de las nuevas viñas respondía a una lógica ligada a su inscripción dentro del sistema de redes de infraestructuras territoriales. Contar con la conjunción de distintas infraestructuras como el ferrocarril, caminos y canales de irrigación

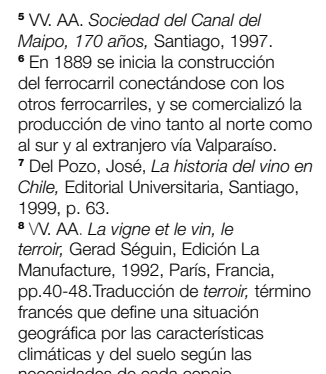

5 W. AA. Sociedad del Canal del
Maipo, 170 años, Santiago, 1997 . ${ }^{6}$ En 1889 se inicia la construcción os ferrocarriles, y se comercializó la producción de vino tanto al norte co Del Pozo, José, La historia del vino en 999, p. 63 AA. La vigne et le vin, le anufacture, 1992, París, Francia, eográfica por las características necesidades de cada cepaje. fue determinante para asegurar el éxito y permanencia en el tiempo de las viñas.

De hecho, el control de los canales de irrigación era esencial para la vitivinicultura, y permitió disponer de un recurso indispensable al cultivo de la vid de manera permanente. Asegurar la cantidad de agua disponible determinaba la extensión de las plantaciones previendo el rendimiento de la explotación. Los caminos y ferrovías eran necesarios para el transporte del vino hasta Santiago para su comercialización.

A otra escala, la división interna de las viñas generó una organización espacial racionalizada, producto de las nuevas técnicas de explotación introducidas por la vitivinicultura moderna.

La organización espacial de las explotaciones puede ser reconocida como un sistema de relaciones espaciales entre dos entidades claramente distintas. Estas dos entidades se diferenciaban por la función que cumplían de la organización productiva. Así se identificaban un área destinada al cultivo y otra a los servicios. La zona de servicio presentaba distintos grados de desarrollo: podía contemplar desde la bodega hasta varios edificios tales como caballerizas, vivienda de inquilinos, una capilla, la residencia de campo del propietario y su parque. Es la organización de la zona de servicio la que señalaba la envergadura de la explotación.

Así, el grado de complejidad interna de la nueva explotación productiva varió según la amplitud y el prestigio del propietario, conservando articulaciones espaciales como características propias a cada viñedo.

El sector de cultivos se encontraba adyacente al canal, del cual obtenía su título de regadío, mientras que la cercanía de los caminos existentes determinaba la ubicación de la zona de servicio y el acceso a la propiedad.

A diferentes escalas del territorio, las explotaciones vitivinícolas se pueden entender como geogramas, motivos concretos inscritos en el espacio geográfico y que configuran la valorización y apropiación del espacio rural a través de la producción de vino.

La bodega vinícola: principal motivo del territorio vitivinícola

No sólo el tamaño y la estructura interna caracterizaron la explotación vitivinícola, sino que, además, el conjunto de recintos necesarios a la producción se formalizó en la bodega, edificio único, distinguible de los antiguos galpones agrícolas, dispersos y polifuncionales.

La bodega vinícola (Fig. 3) fue la manifestación arquitectónica de la reforma de la vitivinicultura chilena, como la propuesta y puesta a punto de 
3 Bodegas viña Santa Carolina,

Macul, Santiag

4 Dibujos de trabajos de la

vendimia, cubería y lagar de

la bodega de viña Cousiño

Macul, 1890, autor desconocido.

Tomada de Donoso, Max,

Cousiño Macul, Huellas de l

familia, ed. privada, Santiago de

Chile, 1999.
5 Cubería y lagar de la bodeg

de viña Santa Carolina, Macul

1910, autor desconocido.

Fotografía tomada de Walton, J.
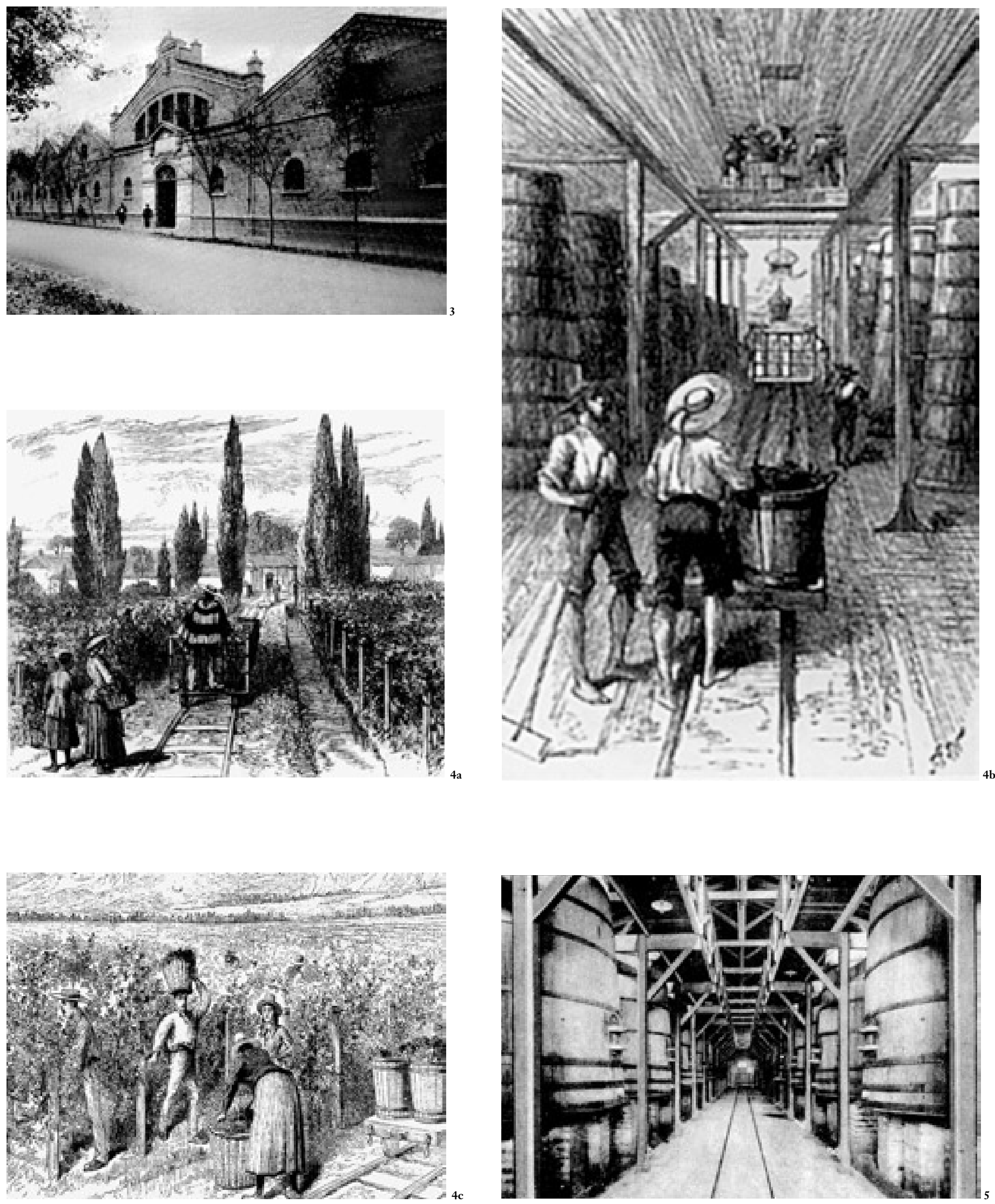
un nuevo programa arquitectónico.

A finales del siglo XIX se publicaron una serie de tratados sobre las técnicas de producción del vino entre los cuales destaca el escrito por Manuel Rojas $^{10}$, que presentó una síntesis del saber en la materia, incluso del programa arquitectónico. El principal aporte de estos tratados estuvo en formular los principios sobre los cuales basarse para resolver la organización espacial de la bodega, diferenciándose de los galpones de almacenamiento, al permitir un control de la calidad en cada uno de los recintos y fases de la crianza del vino. En esta época la bodega vinícola fue considerada como un solo edificio constituido por distintos locales, especificados y articulados según las necesidades de la elaboración del vino. La producción de vino a partir de la uva (vinificación) requiere tres etapas, a las cuales corresponden locales diferenciados por sus características dimensionales y ambientales. La primera etapa de la vinificación consiste en reducir la uva a mosto, en un recinto denominado lagar (Fig. 4, 5, 6 y 7), donde la uva es prensada vertiendo su jugo y el orujo en grandes cubas, donde fermenta. Una vez finalizada la fermentación se trasiega el mosto a vasijas para ser controlado el primer año en la bodega de crianza (Fig. 8 y 9). La última etapa de la crianza es el envejecimiento y sucede en una bodega de guarda (Fig. 10 y 11), donde permanece el vino hasta antes de ser embotellado. Todos los locales eran dimensionados en relación con cuatro variables definidas por el enólogo: el rendimiento de la cepa, la extensión del viñedo y las dimensiones de las vasijas vinarias y su disposición al interior de los locales. Las medidas del edificio reflejan entonces la amplitud de la explotación.

El lagar y la cubería, por estar vinculados funcionalmente, estaban dispuestos el uno sobre la otra, en un solo recinto, siendo el lagar un piso técnico sobre la cubería. El recinto que los contenía debía ser bien ventilado y la temperatura mantenerse entre 15 y 20 grados. La bodega de crianza debía estar en relación directa con la cubería, además de ser oscura, seca, sin corriente de aire y mantener una temperatura entre $12 \mathrm{y}$ 18 grados. De la misma manera, la bodega de guarda debía estar relacionada con la bodega de crianza, además de ser oscura, seca, aislada de la influencia exterior con posibilidades de aireación, manteniendo una temperatura entre 10 y 15 grados.

En la configuración de la planta como en la del corte, la distribución de los recintos obedecía, por un lado a la circulación del producto en sus distintos estados -desde la uva hasta el vino- y por otro lado por las necesidades ambientales de cada uno de ellos. Así, el lagar y la cubería se ubicaban en el costado del edificio que daba hacia el viñedo; la bodega de crianza era adyacente, fuese paralela o en escuadra; y la bodega de guarda por debajo de ésta, en subterráneo. La organización espacial estaba regida por la necesidad de accesibilidad al vino para su desplazamiento y su control de calidad.

Así, el pasillo, como estructura espacial, se convirtió en una característica particular de las nuevas bodegas, siendo la resultante de todos los desplazamientos de las personas y del vino.

En términos materiales, la mayoría de los ejemplos de bodegas conocidas estaba construida en adobe, albañilería en ladrillo cocido y mampostería en piedra presentando dos sistemas constructivos: el muro asociado a cañones corridos y el pilar asociado a bóvedas de aristas, de lo cual resultaban dos características espaciales: recintos compartimentados o naves comunicadas.

Las bodegas vinícolas manifestaron la sistematización de los procesos productivos y la eficiencia de los desplazamientos interiores. A partir de la articulación de las dimensiones del programa se estableció el diseño de una forma arquitectónica claramente legible en la volumetría exterior.

\section{Un cuadro vitivinícola como configuración} de paisaje.

El paisaje construido por la reforma de la vitivinicultura, en el Llano del Maipo, se presenta como la imagen resultante de los adelantos permitidos por diversas técnicas y su implementación en el territorio.

Fue, por lo tanto, la aparición de una nueva imagen en el espacio rural, constituida por motivos identificables y recurrentes que inauguró la construcción del paisaje del vino.

Al volver nuestra mirada sobre la imagen de la viña Panquehue, su estructura nos evoca el paisaje del vino, que se sustenta sobre la organización espacial de una explotación agrícola. Esta imagen articula configuraciones materiales específicas y visibles, que son entendidas no como elementos aislados sino que articulados entre sí como motivos paisajísticos.

Como telón de fondo, la hilera de álamos que acompaña los canales en todo el valle identifica el control del agua, como el tren, la posibilidad de transporte e intercambio del vino. Otro ejemplo como el clos, materializado por la pirca, se reconoce como el cierro perimetral de las viñas. La ordenada orientación de las melgas produce un patrón visual de líneas paralelas que,
${ }^{10}$ Rojas Manuel, Tratado de Viticultura yvinificación Santiago, Chile, 1897 . Manuel Rojas era ingeniero agrícola director de la Escuela de Agricultura de Talca. En 1897 fue director de la Escuela de Vitivinicultura de Cauquenes. En 1901 aparece dentro de la planta de
profesores del Instituto Agricola de la Quinta Normal de Agricultura. Además, se pueden consultar los dos folletos evinicultura de la Quinta Normal

redactados por René Le
11 W. AA. Lire l'espace, Forme dans "1. AA. Lire lespace, Forme dans Augustin Berque, Ediciones Ed Ous colección Reccueil, Bruxelles, 1996, pp. 329-338. agrupadas en cuadras separadas, por caminos ortogonales que se fugan, caracterizan el paisaje del vino por una racionalización del espacio productivo a través de un orden geométrico.

En el primer plano aparece la bodega, como la manifestación arquitectónica de la reforma de la vitivinicultura. El tamaño del edificio, la repetición de sus naves, sus fachadas casi ciegas, y la repetición de los frontis destacan la bodega de los antiguos edificios agrícolas y remiten a una imagen edilicia. En esta imagen la bodega toma presencia como el edificio construido que identifica la producción de vino y el control en términos productivos del territorio que lo sustenta. Tomando distancia del conjunto de elementos que componen la imagen, se produce un contraste que hace visibles dos maneras antagónicas de organizar la propiedad, distinguiéndose claramente sus dimensiones espaciales: la productiva y la ornamental.

El orden productivo, caracterizado por un patrón gráfico, geometrizado, repetitivo y extensible, se contrapone al orden ornamental del parque, que rompe con la repetición, presentando diversas agrupaciones vegetales, constituyendo un mundo interior, acotado y aislado de la producción. En estos parques las circulaciones curvas y fluidas renuevan el motivo paisajístico, proponiendo una sucesión de vistas y composiciones ornamentales entregadas a la contemplación.

El cuadro del paisaje rural vitivinícola, de finales del siglo XIX, resultó de operaciones técnicas destinadas a racionalizar el espacio rural y a la vez ordenó la imagen del territorio en un conjunto significativo. Este doble proceso fijó en nuestra cultura una identidad del paisaje del vino que ha permanecido en el tiempo.

El cuadro de la viña Panquehue representa entonces más que una prueba de la existencia de un paisaje del vino, pues nos permite leer tanto formas en el espacio como formas en el tiempo ${ }^{\mathbf{1 1}}$. $\mathrm{Al}$ leer este cuadro, a pesar de los 100 años que han pasado desde que fue pintado, se nos revela hoy el sentido del paisaje del vino, éste de hace 100 años, como el que se está construyendo hoy en día con otros motivos.

Estas consideraciones nos sugieren preguntarnos: ¿Cuál es el orden espacial concreto que presentan los viñedos actuales?, ¿cómo éstos se representan hoy en día? y ¿en qué motivos concretos y simbólicos podemos hoy encontrar el sentido del paisaje del vino? Se trataría entonces de construir un punto de vista que permita considerar el territorio del vino como la configuración de motivos que en sus relaciones definen el nuevo paisaje del vino asumiendo la dualidad del término paisaje. ARQ 


\begin{tabular}{|c|c|c|c|}
\hline $\begin{array}{l}6 \text { Cubería y lagar de la viña } \\
\text { Cousiño Macul, 910, autor } \\
\text { desconocido. Fotografía tomada } \\
\text { de Donoso, Max, op.cit. } \\
7 \text { Perspectiva de cubería y lagar } \\
\text { de la viña Lynch - Bages, en } \\
\text { Paulliac. Publicada en Dethir, } \\
\text { Jean, Histoire des vins de Bordeaux, } \\
\text { Ed. Garonne, París, } 1992\end{array}$ & $\begin{array}{l}8 \text { Bodega de crianza de la viña } \\
\text { Santa Rita, } 1930 . \\
\text { Fotografía tomada de Del Pozo, } \\
\text { José, La bistoria del vino en } \\
\text { Cbile, Editorial Universitaria, } \\
\text { Santiago de Chile, } 1999 \\
9 \text { Bodega de crianza de la viña } \\
\text { Lontué, 1930. Fotografía } \\
\text { tomada de Del Pozo, José, } \\
\text { op. cit. }\end{array}$ & $\begin{array}{l}10 \text { Bodega de guarda de la viña } \\
\text { Cousiño Macul, } 1910 . \\
\text { Fotografía tomada de } \\
\text { Poirier, Eduardo, Cbile en } \\
\text { 1910, Edición del Centenario } \\
\text { de la Independencia, } \\
\text { Imprenta Barcelona, } \\
\text { Santiago de Chile, } 1910\end{array}$ & $\begin{array}{l}11 \text { Bodega de guarda de la viña } \\
\text { Santa Carolina, } 1919 . \\
\text { Fotografía tomada de } \\
\text { Walton, J., op.cit. }\end{array}$ \\
\hline
\end{tabular}
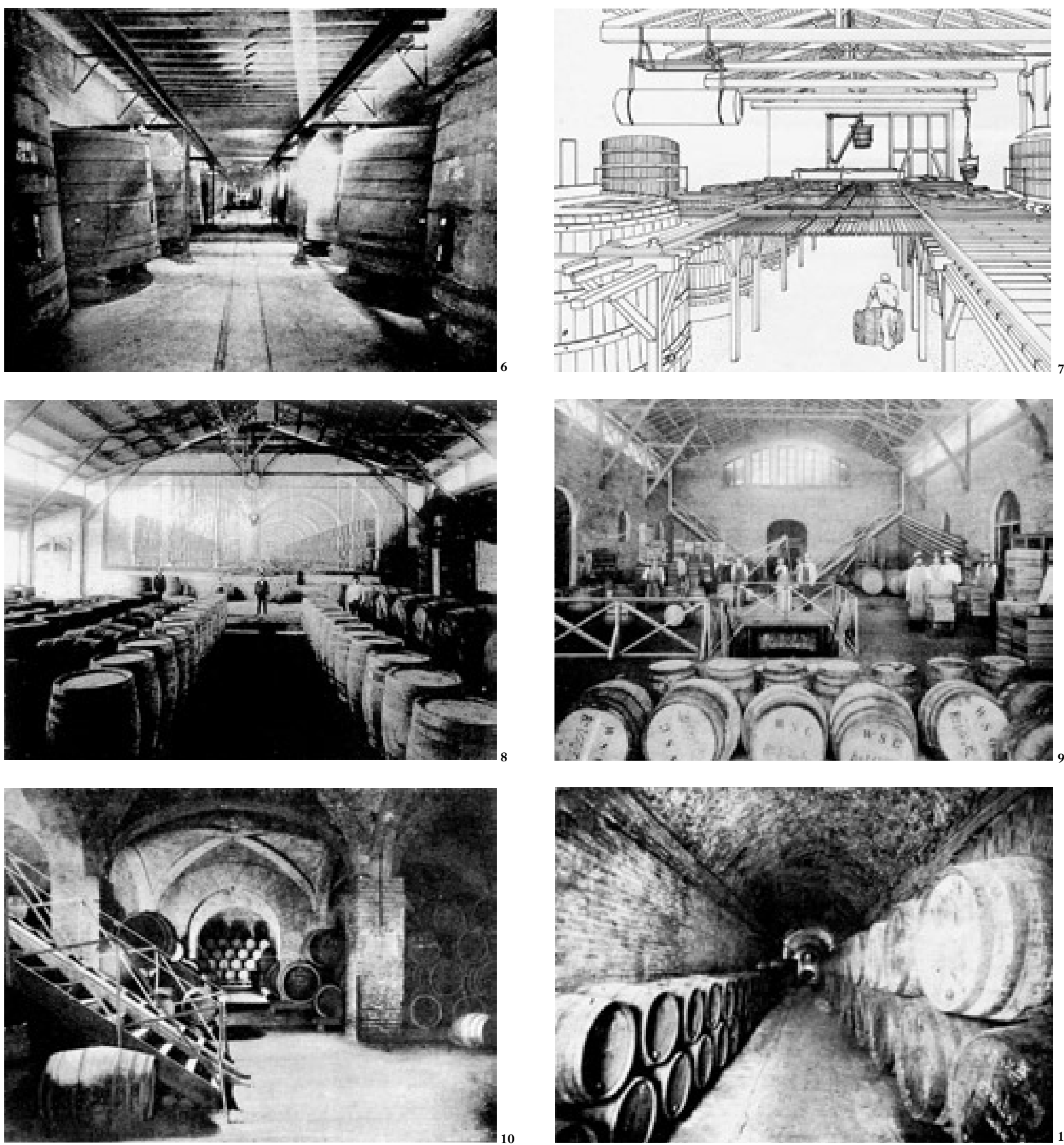